EUROPEAN LABORATORY FOR PARTICLE PHYSICS

CERN-EP/98-038

6 March 1998

\title{
Determination of $A_{\mathrm{FB}}^{b}$ using Jet Charge Measurements in $\mathrm{Z}$ Decays
}

\author{
The ALEPH Collaboration
}

\begin{abstract}
An improved measurement of the forward-backward asymmetry in $\mathrm{Z} \rightarrow b \bar{b}$ decays is presented, based on a sample of 4.1 million hadronic $\mathrm{Z}$ decays collected by ALEPH between 1991 and 1995. Data are analysed as a function of polar angle of the event axis and $b$ purity. The event tagging efficiency and mean $b$-jet hemisphere charge are measured directly from data. From the measured forward-backward jet charge asymmetry, the $b$ quark asymmetry at $\sqrt{s}=m_{\mathrm{Z}}$ is determined to be : $A_{\mathrm{FB}}^{b}=0.1017 \pm 0.0038$ (stat.) \pm 0.0032 (syst.). In the context of the Standard Model this corresponds to a value of the effective weak mixing angle of $\sin ^{2} \theta_{\mathrm{W}}^{\mathrm{eff}}=0.23109 \pm 0.00096$.
\end{abstract}

(To be submitted to Physics Letters B) 


\section{The ALEPH Collaboration}

R. Barate, D. Buskulic, D. Decamp, P. Ghez, C. Goy, J.-P. Lees, A. Lucotte, E. Merle, M.-N. Minard, J.-Y. Nief, B. Pietrzyk

Laboratoire de Physique des Particules (LAPP), IN $P^{3}$-CNRS, F-74019 Annecy-le-Vieux Cedex, France

R. Alemany, G. Boix, M.P. Casado, M. Chmeissani, J.M. Crespo, M. Delfino, E. Fernandez, M. Fernandez-Bosman, Ll. Garrido, ${ }^{15}$ E. Graugès, A. Juste, M. Martinez, G. Merino, R. Miquel, Ll.M. Mir, I.C. Park, A. Pascual, J.A. Perlas, I. Riu, F. Sanchez

Institut de Física d'Altes Energies, Universitat Autònoma de Barcelona, E-08193 Bellaterra (Barcelona), $\operatorname{Spain}^{7}$

A. Colaleo, D. Creanza, M. de Palma, G. Gelao, G. Iaselli, G. Maggi, M. Maggi, S. Nuzzo, A. Ranieri, G. Raso, F. Ruggieri, G. Selvaggi, L. Silvestris, P. Tempesta, A. Tricomi, ${ }^{3}$ G. Zito

Dipartimento di Fisica, INFN Sezione di Bari, I-70126 Bari, Italy

X. Huang, J. Lin, Q. Ouyang, T. Wang, Y. Xie, R. Xu, S. Xue, J. Zhang, L. Zhang, W. Zhao Institute of High-Energy Physics, Academia Sinica, Beijing, The People's Republic of China ${ }^{8}$

D. Abbaneo, U. Becker, P. Bright-Thomas, D. Casper, M. Cattaneo, V. Ciulli, G. Dissertori, H. Drevermann, R.W. Forty, M. Frank, R. Hagelberg, J.B. Hansen, J. Harvey, P. Janot, B. Jost, I. Lehraus, P. Mato, A. Minten, L. Moneta, ${ }^{21}$ A. Pacheco, J.-F. Pusztaszeri, ${ }^{23}$ F. Ranjard, L. Rolandi, D. Rousseau, D. Schlatter, M. Schmitt, ${ }^{20}$ O. Schneider, W. Tejessy, F. Teubert, I.R. Tomalin, H. Wachsmuth

European Laboratory for Particle Physics (CERN), CH-1211 Geneva 23, Switzerland

Z. Ajaltouni, F. Badaud, G. Chazelle, O. Deschamps, A. Falvard, C. Ferdi, P. Gay, C. Guicheney, P. Henrard, J. Jousset, B. Michel, S. Monteil, J-C. Montret, D. Pallin, P. Perret, F. Podlyski, J. Proriol, P. Rosnet

Laboratoire de Physique Corpusculaire, Université Blaise Pascal, IN ${ }^{2} P^{3}$-CNRS, Clermont-Ferrand, F-63177 Aubière, France

J.D. Hansen, J.R. Hansen, P.H. Hansen, B.S. Nilsson, B. Rensch, A. Wäänänen

Niels Bohr Institute, DK-2100 Copenhagen, Denmark ${ }^{9}$

G. Daskalakis, A. Kyriakis, C. Markou, E. Simopoulou, I. Siotis, A. Vayaki

Nuclear Research Center Demokritos (NRCD), GR-15310 Attiki, Greece

A. Blondel, G. Bonneaud, J.-C. Brient, P. Bourdon, A. Rougé, M. Rumpf, A. Valassi, ${ }^{6}$ M. Verderi, H. Videau

Laboratoire de Physique Nucléaire et des Hautes Energies, Ecole Polytechnique, IN ${ }^{2} P^{3}-C N R S$, F-91128 Palaiseau Cedex, France

E. Focardi, G. Parrini, K. Zachariadou

Dipartimento di Fisica, Università di Firenze, INFN Sezione di Firenze, I-50125 Firenze, Italy

M. Corden, C. Georgiopoulos, D.E. Jaffe

Supercomputer Computations Research Institute, Florida State University, Tallahassee, FL 32306-4052, $U S A^{13,14}$

A. Antonelli, G. Bencivenni, G. Bologna, ${ }^{4}$ F. Bossi, P. Campana, G. Capon, F. Cerutti, V. Chiarella,

G. Felici, P. Laurelli, G. Mannocchi, ${ }^{5}$ F. Murtas, G.P. Murtas, L. Passalacqua, M. Pepe-Altarelli

Laboratori Nazionali dell'INFN (LNF-INFN), I-00044 Frascati, Italy

L. Curtis, A.W. Halley, J.G. Lynch, P. Negus, V. O'Shea, C. Raine, J.M. Scarr, K. Smith, P. Teixeira-Dias, A.S. Thompson, E. Thomson

Department of Physics and Astronomy, University of Glasgow, Glasgow G12 8QQ,United Kingdom ${ }^{10}$

O. Buchmüller, S. Dhamotharan, C. Geweniger, G. Graefe, P. Hanke, G. Hansper, V. Hepp, E.E. Kluge, A. Putzer, J. Sommer, K. Tittel, S. Werner, M. Wunsch

Institut für Hochenergiephysik, Universität Heidelberg, D-69120 Heidelberg, Germany ${ }^{16}$ 
R. Beuselinck, D.M. Binnie, W. Cameron, P.J. Dornan, ${ }^{2}$ M. Girone, S. Goodsir, E.B. Martin, N. Marinelli, A. Moutoussi, J. Nash, J.K. Sedgbeer, P. Spagnolo, M.D. Williams

Department of Physics, Imperial College, London SW7 2BZ, United Kingdom ${ }^{10}$

V.M. Ghete, P. Girtler, E. Kneringer, D. Kuhn, G. Rudolph

Institut für Experimentalphysik, Universität Innsbruck, A-6020 Innsbruck, Austria ${ }^{18}$

A.P. Betteridge, C.K. Bowdery, P.G. Buck, P. Colrain, G. Crawford, A.J. Finch, F. Foster, G. Hughes, R.W.L. Jones, M.I. Williams

Department of Physics, University of Lancaster, Lancaster LA1 4YB, United Kingdom ${ }^{10}$

I. Giehl, A.M. Greene, C. Hoffmann, K. Jakobs, K. Kleinknecht, G. Quast, B. Renk, E. Rohne, H.-G. Sander, P. van Gemmeren, C. Zeitnitz

Institut für Physik, Universität Mainz, D-55099 Mainz, Germany ${ }^{16}$

J.J. Aubert, C. Benchouk, A. Bonissent, G. Bujosa, J. Carr, ${ }^{2}$ P. Coyle, F. Etienne, O. Leroy, F. Motsch, P. Payre, M. Talby, A. Sadouki, M. Thulasidas, K. Trabelsi

Centre de Physique des Particules, Faculté des Sciences de Luminy, IN ${ }^{2} P^{3}$-CNRS, F-13288 Marseille, France M. Aleppo, M. Antonelli, F. Ragusa

Dipartimento di Fisica, Università di Milano e INFN Sezione di Milano, I-20133 Milano, Italy

R. Berlich, W. Blum, V. Büscher, H. Dietl, G. Ganis, H. Kroha, G. Lütjens, C. Mannert, W. Männer, H.-G. Moser, S. Schael, R. Settles, H. Seywerd, H. Stenzel, W. Wiedenmann, G. Wolf

Max-Planck-Institut für Physik, Werner-Heisenberg-Institut, D-80805 München, Germany ${ }^{16}$

J. Boucrot, O. Callot, S. Chen, A. Cordier, M. Davier, L. Duflot, J.-F. Grivaz, Ph. Heusse,

A. Höcker, A. Jacholkowska, D.W. Kim, ${ }^{12}$ F. Le Diberder, J. Lefrançois, A.-M. Lutz, M.-H. Schune,

E. Tournefier, J.-J. Veillet, I. Videau, D. Zerwas

Laboratoire de l'Accélérateur Linéaire, Université de Paris-Sud, $I N^{2} P^{3}$-CNRS, F-91405 Orsay Cedex, France

P. Azzurri, G. Bagliesi ${ }^{2}$ G. Batignani, S. Bettarini, T. Boccali, C. Bozzi, G. Calderini, M. Carpinelli, M.A. Ciocci, R. Dell'Orso, R. Fantechi, I. Ferrante, L. Foà, ${ }^{1}$ F. Forti, A. Giassi, M.A. Giorgi, A. Gregorio, F. Ligabue, A. Lusiani, P.S. Marrocchesi, A. Messineo, F. Palla, G. Rizzo, G. Sanguinetti, A. Sciabà, G. Sguazzoni, R. Tenchini, G. Tonelli, ${ }^{19}$ C. Vannini, A. Venturi, P.G. Verdini

Dipartimento di Fisica dell'Università, INFN Sezione di Pisa, e Scuola Normale Superiore, I-56010 Pisa, Italy

G.A. Blair, L.M. Bryant, J.T. Chambers, M.G. Green, T. Medcalf, P. Perrodo, J.A. Strong, J.H. von Wimmersperg-Toeller

Department of Physics, Royal Holloway \& Bedford New College, University of London, Surrey TW20 OEX, United Kingdom ${ }^{10}$

D.R. Botterill, R.W. Clifft, T.R. Edgecock, S. Haywood, P.R. Norton, J.C. Thompson, A.E. Wright

Particle Physics Dept., Rutherford Appleton Laboratory, Chilton, Didcot, Oxon OX11 OQX, United Kingdom $^{10}$

B. Bloch-Devaux, P. Colas, S. Emery, W. Kozanecki, E. Lançon, ${ }^{2}$ M.-C. Lemaire, E. Locci, P. Perez, J. Rander, J.-F. Renardy, A. Roussarie, J.-P. Schuller, J. Schwindling, A. Trabelsi, B. Vallage

CEA, DAPNIA/Service de Physique des Particules, CE-Saclay, F-91191 Gif-sur-Yvette Cedex, France ${ }^{17}$

S.N. Black, J.H. Dann, R.P. Johnson, H.Y. Kim, N. Konstantinidis, A.M. Litke, M.A. McNeil, G. Taylor

Institute for Particle Physics, University of California at Santa Cruz, Santa Cruz, CA 95064, USA ${ }^{22}$

C.N. Booth, C.A.J. Brew, S. Cartwright, F. Combley, M.S. Kelly, M. Lehto, J. Reeve, L.F. Thompson

Department of Physics, University of Sheffield, Sheffield S3 7RH, United Kingdom ${ }^{10}$ 
K. Affholderbach, A. Böhrer, S. Brandt, G. Cowan, C. Grupen, P. Saraiva, L. Smolik, F. Stephan

Fachbereich Physik, Universität Siegen, D-57068 Siegen, Germany ${ }^{16}$

M. Apollonio, L. Bosisio, R. Della Marina, G. Giannini, B. Gobbo, G. Musolino

Dipartimento di Fisica, Università di Trieste e INFN Sezione di Trieste, I-34127 Trieste, Italy

J. Rothberg, S. Wasserbaech

Experimental Elementary Particle Physics, University of Washington, WA 98195 Seattle, U.S.A.

S.R. Armstrong, E. Charles, P. Elmer, D.P.S. Ferguson, Y. Gao, S. González, T.C. Greening, O.J. Hayes, H. Hu, S. Jin, P.A. McNamara III, J.M. Nachtman, ${ }^{24}$ J. Nielsen, W. Orejudos, Y.B. Pan, Y. Saadi, I.J. Scott, J. Walsh, Sau Lan Wu, X. Wu, G. Zobernig

Department of Physics, University of Wisconsin, Madison, WI 53706, USA ${ }^{11}$

\footnotetext{
${ }^{1}$ Now at CERN, 1211 Geneva 23, Switzerland.

${ }^{2}$ Also at CERN, 1211 Geneva 23, Switzerland.

${ }^{3}$ Also at Dipartimento di Fisica, INFN, Sezione di Catania, Catania, Italy.

${ }^{4}$ Also Istituto di Fisica Generale, Università di Torino, Torino, Italy.

${ }^{5}$ Also Istituto di Cosmo-Geofisica del C.N.R., Torino, Italy.

${ }^{6}$ Supported by the Commission of the European Communities, contract ERBCHBICT941234.

${ }^{7}$ Supported by CICYT, Spain.

${ }^{8}$ Supported by the National Science Foundation of China.

${ }^{9}$ Supported by the Danish Natural Science Research Council.

${ }^{10}$ Supported by the UK Particle Physics and Astronomy Research Council.

${ }^{11}$ Supported by the US Department of Energy, grant DE-FG0295-ER40896.

${ }^{12}$ Permanent address: Kangnung National University, Kangnung, Korea.

${ }^{13}$ Supported by the US Department of Energy, contract DE-FG05-92ER40742.

${ }^{14}$ Supported by the US Department of Energy, contract DE-FC05-85ER250000.

${ }^{15}$ Permanent address: Universitat de Barcelona, 08208 Barcelona, Spain.

${ }^{16}$ Supported by the Bundesministerium für Bildung, Wissenschaft, Forschung und Technologie, Germany.

${ }^{17}$ Supported by the Direction des Sciences de la Matière, C.E.A.

${ }^{18}$ Supported by Fonds zur Förderung der wissenschaftlichen Forschung, Austria.

${ }^{19}$ Also at Istituto di Matematica e Fisica, Università di Sassari, Sassari, Italy.

${ }^{20}$ Now at Harvard University, Cambridge, MA 02138, U.S.A.

${ }^{21}$ Now at University of Geneva, 1211 Geneva 4, Switzerland.

${ }^{22}$ Supported by the US Department of Energy, grant DE-FG03-92ER40689.

${ }^{23}$ Now at School of Operations Research and Industrial Engineering, Cornell University, Ithaca, NY 14853-3801, U.S.A.

${ }^{24}$ Now at University of California at Los Angeles (UCLA), Los Angeles, CA 90024, U.S.A.
} 


\section{Introduction}

With the completion of LEP 1 running at energies close to the $\mathrm{Z}$ resonance, ALEPH has accumulated a data sample containing approximately 4.1 million hadronic $\mathrm{Z}$ decays, recorded with precise three-dimensional tracking information. These data offer the opportunity to test the Standard Model [1] predictions of electroweak radiative corrections with improved precision and thus constrain the allowed range of Higgs masses within the model. Hence it is of interest to study channels which offer the greatest sensitivity to electroweak parameters and to develop experimental techniques which make optimal use of the event information in the data set.

One such measurement is the forward-backward asymmetry of $b$ quarks from $Z \rightarrow b \bar{b}$ production. The asymmetry is defined using the angle, $\theta$, between the incoming electron and the outgoing $b$-quark to denote the forward $(\cos \theta>0)$ and backward $(\cos \theta<0)$ hemispheres :

$$
A_{\mathrm{FB}}^{b}=\frac{\sigma_{\mathrm{F}}^{b}-\sigma_{\mathrm{B}}^{b}}{\sigma_{\mathrm{F}}^{b}+\sigma_{\mathrm{B}}^{b}} .
$$

After corrections for acceptance, detector effects, photon exchange and for QED and QCD radiation, the ratio of Standard Model $\mathrm{Z}$ couplings and effective weak mixing angle can be extracted.

Measurement of the asymmetry requires the selection of $b \bar{b}$ events and measurement of the original quark charges. Exploiting the large mass and lifetime of the $b$ quark, it has been shown that such distinctions can be made using a combination [2, 3, 4] of lifetime tagging [5], and a hemisphere charge method [6]. The method of [2] is improved upon here by extending the angular acceptance and analysing data as a function of $b$ quark purity and polar angle. Systematic uncertainties are controlled by use of light quark charge separations determined in an untagged jet charge analysis [7] and simultaneously fitting for both the $b$ hemisphere charge separation, $\delta_{b}$, and the sample $b$ purity, $\mathcal{P}_{b}$. These improvements result in a measurement with enhanced statistical precision and reduced systematic uncertainties arising from the sample flavour composition and use of the hemisphere charge method.

\section{Principles of the Method}

Measurement of $A_{\mathrm{FB}}^{b}$ requires an estimate of the direction of the final state $b$ quark from the decay of the $\mathrm{Z}$ and knowledge of the event sample composition and average charges of jets originating from different quark flavours. The $b \bar{b}$ event axis is estimated using the reconstructed thrust axis, $\vec{T}$, which is orientated to point in the forward direction. The forward-backward orientation of the $b$ quark is determined using the hemisphere charge method [6]. This is inspired by the premise that the electric charge of particles produced during hadronisation of a quark retains some knowledge of the parent quark's charge [8]. Each event is divided into hemispheres by a plane perpendicular to the thrust axis. Hemisphere charges are formed using a summation over particle charges, $q$, weighted by their momentum, $\vec{p}$. The forward hemisphere charge, $Q_{\mathrm{F}}$, is given by :

$$
Q_{\mathrm{F}}=\frac{\sum_{i}^{\overrightarrow{p_{i}} \cdot \vec{T}>0}\left|\overrightarrow{p_{i}} \cdot \vec{T}\right|^{\kappa} q_{i}}{\sum_{i}^{\overrightarrow{p_{i}} \cdot \vec{T}>0}\left|\overrightarrow{p_{i}} \cdot \vec{T}\right|^{\kappa}},
$$

and analogously for $Q_{\mathrm{B}}$, summing over particles with $\overrightarrow{p_{i}} \cdot \vec{T} \leq 0$. The parameter $\kappa$ is used to optimise the measurement sensitivity and provides a cross-check of the method, as results determined with different values of $\kappa$ must be consistent. On average, the charges of hemispheres containing the quark, $Q_{f}$, and the antiquark, $Q_{\bar{f}}$, prior to gluon radiation, differ by the quantity :

$$
\delta_{f}=\left\langle Q_{f}-Q_{\bar{f}}\right\rangle
$$

in $f \bar{f}$ events. This is referred to as the charge separation for flavour $f$. A quark asymmetry, $A_{\mathrm{FB}}^{f}$, for flavour $f$ is then proportional to the mean charge flow, $\left\langle Q_{\mathrm{FB}}^{f}\right\rangle$, between forward and 
backward hemispheres :

$$
\left\langle Q_{\mathrm{FB}}^{f}\right\rangle=\left\langle Q_{\mathrm{F}}^{f}-Q_{\mathrm{B}}^{f}\right\rangle=\delta_{f} A_{\mathrm{FB}}^{f},
$$

in pure $f \bar{f}$ events. As shown in [2], the same sample of events used to measure $\left\langle Q_{\mathrm{FB}}^{f}\right\rangle$ can also be used to extract $\delta_{f}$. This may be understood by considering a single hemisphere charge measurement, $Q_{f}$, which can be written as :

$$
Q_{f}=\frac{\delta_{f}}{2}+\mathcal{R}_{f} \text { or } Q_{\bar{f}}=-\frac{\delta_{f}}{2}+\mathcal{R}_{\bar{f}}
$$

where $\mathcal{R}$ represents measurement fluctuations due to fragmentation and detector effects. The product of the two hemisphere charges then averages to :

$$
\left\langle Q_{f} Q_{\bar{f}}\right\rangle=\left\langle Q_{\mathrm{F}} Q_{\mathrm{B}}\right\rangle=\frac{-\delta_{f}^{2}}{4}+\left\langle\mathcal{R}_{f} \mathcal{R}_{\bar{f}}\right\rangle
$$

given that $\mathcal{R}_{f}-\mathcal{R}_{\bar{f}}$ averages to zero. The measurement fluctuations are correlated, ie. $\left\langle\mathcal{R}_{f} \mathcal{R}_{\bar{f}}\right\rangle \neq$ 0 . This is due to effects of charge conservation, sharing a common event axis and crossover of particles close to the hemisphere boundary. The correlation is small and insensitive to the details of fragmentation. The effects of secondary interactions in the detector material can be estimated [7] from the total charge, $\left\langle Q^{f}\right\rangle$, which is measured in data :

$$
\left\langle Q^{f}\right\rangle=\left\langle Q_{\mathrm{F}}^{f}+Q_{\mathrm{B}}^{f}\right\rangle .
$$

The charge separation can be extracted from data using the measured quantity, $\bar{\delta}_{f}$ :

$$
\bar{\delta}_{f}^{2}=-4\left\langle Q_{\mathrm{F}}^{f} Q_{\mathrm{B}}^{f}\right\rangle-\left\langle Q_{\mathrm{FB}}^{f}\right\rangle^{2}+\left\langle Q^{f}\right\rangle^{2} .
$$

This tracks effects of fragmentation or decay model uncertainties on the determination of $\delta_{f}$ and has negligible sensitivity to the electroweak asymmetry or charge biases from secondary interactions [7]. It is related to $\delta_{f}$ by :

$$
\begin{aligned}
\bar{\delta}_{f}^{2} & =\delta_{f}^{2}-4\left\langle\mathcal{R}_{f} \mathcal{R}_{\bar{f}}\right\rangle-\left\langle Q_{\mathrm{FB}}^{f}\right\rangle^{2}+\left\langle Q^{f}\right\rangle^{2} \\
& =\left[\delta_{f}\left(1+k_{f}\right)\right]^{2},
\end{aligned}
$$

where both $\left\langle Q_{\mathrm{FB}}^{f}\right\rangle$ and $\left\langle Q^{f}\right\rangle$ are small and measurable in data. Consequently, $\bar{\delta}_{f}$ is equal to $\delta_{f}$, to within a $\kappa$-dependent correction factor, $k_{f}$, which is $\sim 18 \%$ for $b$ quarks at $\kappa=0.5$ and decreases rapidly for higher $\kappa$ values.

The quantities, $\bar{\delta},\left\langle Q_{\mathrm{FB}}\right\rangle$ and $\langle Q\rangle$, are measured directly in subsamples of hadronic $\mathrm{Z}$ decays in which the fraction of $b \bar{b}$ events is increased by tagging the decay products of long-lived particles. The quantity, $P_{\text {hem }}$, is evaluated as the probability that a hemisphere arises from $u, d$ or $s$ quark production, and is based upon the observed impact parameters of charged tracks in that hemisphere. Smaller values of $P_{\text {hem }}$ correspond to enriched $b$ quark samples [5]. Events are classified according to the smallest of the two hemisphere probabilities, $P_{\mathrm{hem}}^{\mathrm{min}}$. The flavour composition of the sample in each bin of $\cos \theta$ and $P_{\mathrm{hem}}^{\min }$ is denoted by the quark flavour purities $\left(\mathcal{P}_{u}, \mathcal{P}_{d}, \mathcal{P}_{s}, \mathcal{P}_{c}, \mathcal{P}_{b}\right)$. Measurement is made of each sample's forward-backward charge asymmetry :

$$
\left\langle Q_{\mathrm{FB}}\right\rangle(\cos \theta)=\sum_{f=u d s c b} \mathcal{P}_{f}(\cos \theta) \delta_{f}(\cos \theta) A_{\mathrm{FB}}^{f}(\cos \theta),
$$

the mean hemisphere charge separation, $\bar{\delta}$ :

$$
\bar{\delta}^{2}=\sum_{f=u d s c b} \mathcal{P}_{f}\left(\bar{\delta}_{f}\right)^{2},
$$


and total event tagging efficiency :

$$
\varepsilon_{\text {total }}=\sum_{f=u d s c b} \mathrm{R}_{f} \varepsilon_{f}
$$

The quantities $\varepsilon_{f}$ and $\mathrm{R}_{f}$ are the event tagging efficiency and fraction of $f \bar{f}$ events respectively. The sample purities in each bin are then calculated from :

$$
\mathcal{P}_{f}=\frac{\mathrm{R}_{f} \varepsilon_{f}}{\varepsilon_{\text {total }}},
$$

where the ratio, $\varepsilon_{f} / \varepsilon_{\text {total }}$, accounts for possible selection biases. The asymmetry, $A_{\mathrm{FB}}^{f}$, in equation (7) depends on $\cos \theta$ according to :

$$
A_{\mathrm{FB}}^{f}(\cos \theta)=\frac{8}{3} A_{\mathrm{FB}}^{f} \frac{\cos \theta}{1+\cos ^{2} \theta},
$$

and similarly, all terms in equations (8), (9) and (10) are functions of $\cos \theta$. The hemisphere charge separation, $\delta_{b}$, is fixed by the $\bar{\delta}$ measurement, knowledge of the hemisphere charge correlation factors, $k_{f}$, defined in equation (6) and light quark separations, $\delta_{u}, \delta_{d}, \delta_{s}$ and $\delta_{c}$. Measurements of $\delta_{c}$ are obtained from samples, enriched using either an impact parameter tag or fast $D^{*}$ from charm decays, as described in [7]. Flavour symmetry principles, constrained by fitting inclusive particle distributions in ALEPH data with fragmentation models and the measured value of $\bar{\delta}$ in the inclusive hadronic sample [7], are used to determine $\delta_{u}, \delta_{d}$ and $\delta_{s}$. The $b$ quark forward-backward asymmetry, $A_{\mathrm{FB}}^{b}$, may then be extracted using :

$$
A_{\mathrm{FB}}^{b}=\frac{1}{\mathcal{P}_{b} C_{b}}\left[\frac{\left\langle Q_{\mathrm{FB}}\right\rangle}{\delta_{b}}-\frac{1}{\delta_{b}} \sum_{f=u d s c} \mathcal{P}_{f} C_{f} \delta_{f} A_{\mathrm{FB}}^{f}\right],
$$

where $C_{f}$ are flavour dependent acceptance factors for the given $\left(\cos \theta, P_{\mathrm{hem}}^{\min }\right)$ bin selected.

\section{The ALEPH detector}

The ALEPH detector and its performance are described in detail elsewhere [9]. Only those features relevant for the current analysis are given here. The tracking system consists of two layers of double-sided silicon vertex-detector (VDET), an inner tracking chamber (ITC) and a time projection chamber (TPC) immersed in an axial magnetic field of $1.5 \mathrm{~T}$. The VDET single hit resolution is $12 \mu \mathrm{m}$ at normal incidence for both the $r \phi$ and $r z$ projections and the polar angle coverage of the inner and outer layers are $|\cos \theta|<0.84$ and $|\cos \theta|<0.69$ respectively. The ITC provides up to $8 r \phi$ hits from 16 to $26 \mathrm{~cm}$ relative to the beam with an average resolution of $150 \mu \mathrm{m}$ and has an angular coverage of $|\cos \theta|<0.97$. The TPC measures up to 21 three-dimensional points per track at radii between 40 and $171 \mathrm{~cm}$, with an $r \phi$ resolution of $170 \mu \mathrm{m}$ and an $r z$ resolution of $740 \mu \mathrm{m}$ and with an angular coverage of 0.97 in $\cos \theta$. Tracks are reconstructed using the TPC, ITC and VDET. A transverse momentum resolution of $\sigma\left(1 / p_{T}\right)=0.0006(\mathrm{GeV} / c)^{-1}$ is observed for $45 \mathrm{GeV}$ muons. Multiple scattering dominates at low momentum and adds a constant term of 0.005 to $\sigma\left(p_{T}\right) / p_{T}$. In hadronic events, the impact parameter resolution, $\sigma_{\delta}$, can be parameterised as, $\sigma_{\delta}=25+95 / p(\mathrm{GeV} / \mathrm{c}) \mu \mathrm{m}$. Surrounding the TPC is the electromagnetic calorimeter (ECAL), consisting of 45 layers of lead interleaved with proportional wire chambers. The ECAL is used to identify photons and electrons and gives an energy resolution $\sigma(E) / E=0.18 / \sqrt{E(\mathrm{GeV})}+0.009$. The hadron calorimeter (HCAL) is formed by the iron of the magnet return yoke interleaved with 23 layers of streamer tubes. The HCAL is used to measure hadronic energy, and acts as part of the muon identification system, with the muon detector. 


\section{Event Selection}

The data set used for this analysis consists of approximately four million hadronic Z decays recorded by ALEPH during the period 1991 to 1995 in a centre-of-mass energy range of $m_{\mathrm{Z}} \pm$ $3 \mathrm{GeV} / c^{2}$. Events are selected according to the standard ALEPH hadronic event selection [5] based on good charged tracks. A good charged track must have a $|\cos \theta|<0.95$ and have at least four TPC coordinates, a distance of closest approach to the interaction point of less than $10 \mathrm{~cm}$ along the beam direction and $2 \mathrm{~cm}$ transverse to it. This selection requires at least five such tracks in the TPC. The sum of the energies of the tracks must be greater than $10 \%$ of the centre of mass energy. This selection has an efficiency of $\sim 97.4 \%$ and introduces no significant flavour bias. The backgrounds from $\mathrm{Z}$ decays to $\tau^{+} \tau^{-}$and $\gamma \gamma$ interactions are estimated to be $\sim 0.2$ and $\sim 0.3 \%$ respectively. Both backgrounds are effectively removed after the application of impact parameter tag cuts, and so are safely neglected.

The average beamspot position is determined every 75 events and used to determine the event-by-event interaction point [5] with a typical precision of $50 \times 10 \times 60 \mu \mathrm{m}^{3}$ in horizontal, vertical and beam directions respectively. For the calculation of the impact parameter tag variables, each event must have at least one good charged track with VDET information and a minimum of two jets with momenta greater than $10 \mathrm{GeV}$ and polar angle greater than $5.7^{\circ}$. The thrust axis is determined in each event from all charged tracks and calorimeter clusters using the ALEPH energy flow package [10]. To ensure the event is well contained within the detector volume, the cosine of the polar angle of the thrust axis must be less than 0.9. The final acceptance of these cuts is $\sim 87 \%$ giving a sample of $3,578,462$ hadronic events for the remainder of the analysis.

Data are analysed as a function of the cosine of the polar angle of the thrust axis and their apparent lifetime, in ranges of $\cos \theta$ and $P_{\mathrm{hem}}^{\mathrm{min}}$. Table 1 shows the $P_{\mathrm{hem}}^{\mathrm{min}}$ binning used and the calculated sample purities together with the predicted fractions of $b \bar{b}$ events determined from the total tagging efficiency. As the $b$ quark content of the first bin is very small, this bin is not used

\begin{tabular}{|c|c|c|c|c|c|c|c|}
\hline \multicolumn{3}{|c|}{$P_{\text {hem }}^{\min }$ bin } & $b$ Purity & $c$ Purity & uds Purity & Fraction of & Fraction of all \\
\hline 1.0 & $\rightarrow$ & 0.1 & $2.34 \%$ & $14.09 \%$ & $83.57 \%$ & $6.27 \%$ & $57.80 \%$ \\
\hline 0.1 & $\rightarrow$ & 0.032 & $13.47 \%$ & $25.61 \%$ & $60.92 \%$ & $8.54 \%$ & $13.66 \%$ \\
\hline 0.032 & $\rightarrow$ & 0.005 & $33.36 \%$ & $31.03 \%$ & $35.61 \%$ & $15.72 \%$ & $10.16 \%$ \\
\hline 0.005 & $\rightarrow$ & 0.001 & $57.56 \%$ & $28.12 \%$ & $14.32 \%$ & $12.36 \%$ & $4.63 \%$ \\
\hline 0.001 & $\rightarrow$ & 0.0001 & $76.97 \%$ & $17.96 \%$ & $5.07 \%$ & $15.24 \%$ & $4.27 \%$ \\
\hline 0.0001 & $\rightarrow$ & 0.000001 & $91.67 \%$ & $7.18 \%$ & $1.14 \%$ & $21.32 \%$ & $5.01 \%$ \\
\hline 0.000001 & $\rightarrow$ & 0.0 & $98.92 \%$ & $0.86 \%$ & $0.22 \%$ & $20.55 \%$ & $4.48 \%$ \\
\hline
\end{tabular}

Table 1: Estimated event purities and fractions of $b \bar{b}$ events, and all events, in each $P_{\mathrm{hem}}^{\min }$ bin.

further in the analysis. Results obtained from each bin of $P_{\mathrm{hem}}^{\min }$ are statistically independent and can be combined. An inclusive selection of $P_{\mathrm{hem}}^{\min }<0.001$ and $\cos \theta \leq 0.9$, would yield an estimated $b \bar{b}$ selection efficiency of $55.7 \%$ and a purity of $89.5 \%$.

Using the fraction of events selected in each $\left(\cos \theta, P_{\text {hem }}^{\min }\right)$ bin, $\epsilon_{\text {total }}$, the sample $b$ purity is determined from :

$$
\mathcal{P}_{b}=1-\frac{\sum_{f=u d s c} \mathrm{R}_{f} \varepsilon_{f}}{\varepsilon_{\text {total }}} .
$$

The nominal set of Standard Model input parameters assumed in the fitting procedure are given in Table 2. The fractions of $f \bar{f}$ events in the sample, $\mathrm{R}_{f}$, are set to the values given in Table 2 which correspond to the Standard Model predictions for $m_{\mathrm{Z}}=91.1866 \mathrm{GeV}, m_{\mathrm{t}}=173.1 \mathrm{GeV}$, $m_{\mathrm{H}}=115 \mathrm{GeV}$ and $\alpha_{\mathrm{s}}=0.120[11]$.

Monte Carlo is used to determine light quark and charm event tag efficiencies, $\varepsilon_{u d s}$ and $\varepsilon_{c}$, 


\begin{tabular}{|c|r|r|r|r|}
\hline Parameter, $\mathbf{P}$ & $\sqrt{s}=89.43 \mathrm{GeV}$ & $\sqrt{s}=91.25 \mathrm{GeV}$ & $\sqrt{s}=92.97 \mathrm{GeV}$ & $\partial A_{\mathrm{FB}}^{b} / \partial \mathbf{P}$ \\
\hline $\mathrm{R}_{u}$ & 0.1740 & 0.1729 & 0.1735 & +0.0082 \\
$\mathrm{R}_{d}$ & 0.2190 & 0.2195 & 0.2192 & -0.0033 \\
$\mathrm{R}_{s}$ & 0.2190 & 0.2195 & 0.2191 & -0.0030 \\
$\mathrm{R}_{c}$ & 0.1733 & 0.1726 & 0.1730 & +0.0859 \\
$\mathrm{R}_{b}$ & 0.2147 & 0.2155 & 0.2151 & -0.0113 \\
\hline$A_{\mathrm{FB}}^{u}$ & -0.0341 & 0.0679 & 0.1301 & +0.0103 \\
$A_{\mathrm{FB}}^{d}$ & 0.0625 & 0.1026 & 0.1270 & -0.0077 \\
$A_{\mathrm{FB}}^{s}$ & 0.0625 & 0.1026 & 0.1271 & -0.0097 \\
$A_{\mathrm{FB}}^{c}$ & -0.0342 & 0.0680 & 0.1304 & +0.0927 \\
\hline
\end{tabular}

Table 2: Summary of Standard Model inputs used in the fitting procedure described in the text and dependencies of the measured $A_{\mathrm{FB}}^{b}$.

together with estimates of their systematic uncertainties. A small disagreement [12] is found between the observed total event tagging efficiencies in data and standard ALEPH Monte Carlo [13]. This is found to be partially due to incorrect modelling of the number of tracks with VDET information, and more significantly because of inadequate modelling of detector material and the track multiple scattering. In order to account for the effect of having too many tracks simulated with VDET information, a small fraction of randomly selected Monte Carlo tracks are removed from the calculation of hemisphere tag probabilities. Differences in modelling of the track resolutions are accounted for by smearing the impact parameter significance of Monte Carlo tracks to bring it into accord with that found in data. These corrections are parameterised in terms of the number of VDET hits, particle momentum and $\cos \theta$ of the charged particle track.

The charm tag efficiency is sensitive to the input charm hadron lifetimes, production crosssections and branching fractions, while both charm and light quark efficiencies are sensitive to the amount of gluon splitting to heavy flavours, $g \rightarrow c \bar{c}$ and $g \rightarrow b \bar{b}$. Consequently, Monte Carlo events are reweighted to reproduce recent experimental measurements of these quantities [12]. These reweighting techniques are also used to estimate systematic errors arising from uncertainties in the flavour composition. Both Monte Carlo correction procedures are described in detail in [12]. Table 3 shows the calculated light quark and charm event tag efficiencies, integrated over $\cos \theta$, with their systematic uncertainties. The $\cos \theta$ dependence of these efficiencies is taken from Monte Carlo simulation.

\begin{tabular}{|c|c|c|c|c|c|c|c|c|}
\hline \multicolumn{3}{|c|}{$P_{\text {hin }}^{\min }$ bin } & \multicolumn{3}{|c|}{$\varepsilon_{u d s}(\%)$} & \multicolumn{3}{|c|}{$\varepsilon_{c}(\%)$} \\
\hline 0.1 & $\rightarrow$ & 0.032 & 13.60 & $\pm 0.62 \pm$ & 0.65 & 20.26 & $\pm 0.91 \pm$ & 0.49 \\
\hline 0.032 & $\rightarrow$ & 0.005 & 5.91 & $\pm 0.27 \pm$ & 0.31 & 18.26 & $\pm 0.65 \pm$ & 0.24 \\
\hline 0.005 & $\rightarrow$ & 0.001 & 1.08 & $\pm 0.07 \pm$ & 0.05 & 7.54 & $\pm 0.28 \pm$ & 0.03 \\
\hline 0.001 & $\rightarrow$ & 0.0001 & 0.35 & $\pm 0.03 \pm$ & 0.01 & 4.44 & $\pm 0.18 \pm$ & 0.05 \\
\hline 0.0001 & $\rightarrow$ & 0.000001 & 0.09 & $\pm 0.01 \pm$ & 0.00 & 2.09 & $\pm 0.10 \pm$ & 0.01 \\
\hline 0.000001 & $\rightarrow$ & 0.0 & 0.02 & $\pm 0.00 \pm$ & 0.00 & 0.22 & $\pm 0.02 \pm$ & 0.00 \\
\hline
\end{tabular}

Table 3: Estimated light quark and charm event tag efficiencies in the reweighted Monte Carlo simulation described in the text. Systematic errors are given, due to the uncertainty in the physics input parameters and detector simulation respectively. Light quark and charm detector simulation errors are $100 \%$ correlated and the physics input errors are slightly correlated via the common uncertainty in the amount of $g \rightarrow c \bar{c}$ and $g \rightarrow b \bar{b}$. 


\section{Charge Asymmetry and Event Tag Measurements}

In each recorded event, the two hemisphere charges are calculated according to equation (1) for 5 different $\kappa$ values of $0.3,0.5,1.0,2.0$ and $\infty$. A $\kappa$ value of $\infty$ corresponds to using only the leading track in each hemisphere. Good charged tracks with a $p_{\mathrm{T}}$ relative to the beam greater than $200 \mathrm{MeV} / \mathrm{c}$ are used. The measured quantities, $\left\langle Q_{\mathrm{FB}}\right\rangle$ and $\bar{\delta}$, are then calculated according to equations (3) and (7) respectively. The $\cos \theta$ distributions for the means of these measured quantities are shown in Figure 1 for each $P_{\text {hem }}^{\min }$ bin. Plots of $\left\langle Q_{\mathrm{FB}}\right\rangle$ and $\bar{\delta}$ are shown for $\kappa=0.5$ and 2.0 only, although all $5 \kappa$ values are used later for the fitted results. Measurements for different $\kappa$ values of $\left\langle Q_{\mathrm{FB}}\right\rangle$ (and $\bar{\delta}$ ) are correlated. Their degree of correlation is determined directly from data. The correlations are large, varying between 50 and $100 \%$ [7].

In the central region of the detector $(\cos \theta<0.6), \bar{\delta}$ is flat and $\left\langle Q_{\mathrm{FB}}\right\rangle$ displays a linear $\cos \theta$ dependence arising from the underlying asymmetry. At high $\cos \theta$, near the edge of the tracking acceptance, the charged track reconstruction deteriorates, causing both $\bar{\delta}$ and $\left|\left\langle Q_{\mathrm{FB}}\right\rangle\right|$ to decrease. The total event tag efficiency is also measured, and decreases as the polar angle approaches the edges of the single and double layer vertex detector acceptance. It is also apparent that the magnitude of the measured charge asymmetry increases with $b$ purity, as the cancellation with the oppositely signed charm charge asymmetry diminishes.

The forward-backward asymmetry, $A_{\mathrm{FB}}^{b}$, is extracted in each of the statistically independent $P_{\text {hem }}^{\min }$ bins. This is done by performing a fit for each $P_{\text {hem }}^{\min }$ bin. The inputs to this fit are the following quantities measured in 9 different bins of $\cos \theta$ :

- $\epsilon_{\text {total }}$ (9 values)

- $\left\langle Q_{\mathrm{FB}}\right\rangle$ for 5 different values of $\kappa(9 \times 5$ values $)$ and

- $\bar{\delta}$ for 5 different values of $\kappa(9 \times 5$ values $)$.

The fit is used to determine :

- the value of $\delta_{b}$ in each bin of $\cos \theta$ and for each value of $\kappa(9 \times 5$ parameters $)$,

- the $b$-purity in each bin of $\cos \theta(9$ parameters $)$ and

- the $b$ quark forward-backward asymmetry, $A_{\mathrm{FB}}^{b}$.

Fit results for the various $P_{\text {hem }}^{\min }$ bins are then compared and combined.

\section{$6 \quad$ Results and Systematic Uncertainties}

The method of extracting the forward-backward asymmetry of the $b$ quark operates separately in each of the statistically independent $P_{\mathrm{hem}}^{\min }$ bins. They are later merged in order to optimise the combined statistical and systematic sensitivity of the measurement. In the fitting procedure, light quark and charm physical asymmetries are set equal to their Standard Model values given in Table 2.

The values for $\delta_{b}$ are extracted from the corresponding measurements of $\bar{\delta}$ according to equations (6) and (8). Systematic uncertainties enter through the hemisphere correlation factors, $k_{f}$, for each quark flavour, which are determined using the JETSET [14] fragmentation model and are shown in Table 4. Systematic uncertainties are estimated by independently varying model parameters and combining observed deviations in quadrature. Lighter quark hemisphere charge separations, $\delta_{u}, \delta_{d}, \delta_{s}$ and $\delta_{c}$ are shown in Table 4 together with their systematic uncertainties.

Charge correlation factors, together with light quark and charm charge separations, are assumed to be independent of $\cos \theta$. Monte Carlo simulation indicates that this assumption is valid to the required precision. The bin-by-bin $b$ purities are determined from measurement of the total event tagging efficiency, $\varepsilon_{\text {total }}$, with background efficiencies taken from Monte Carlo so 

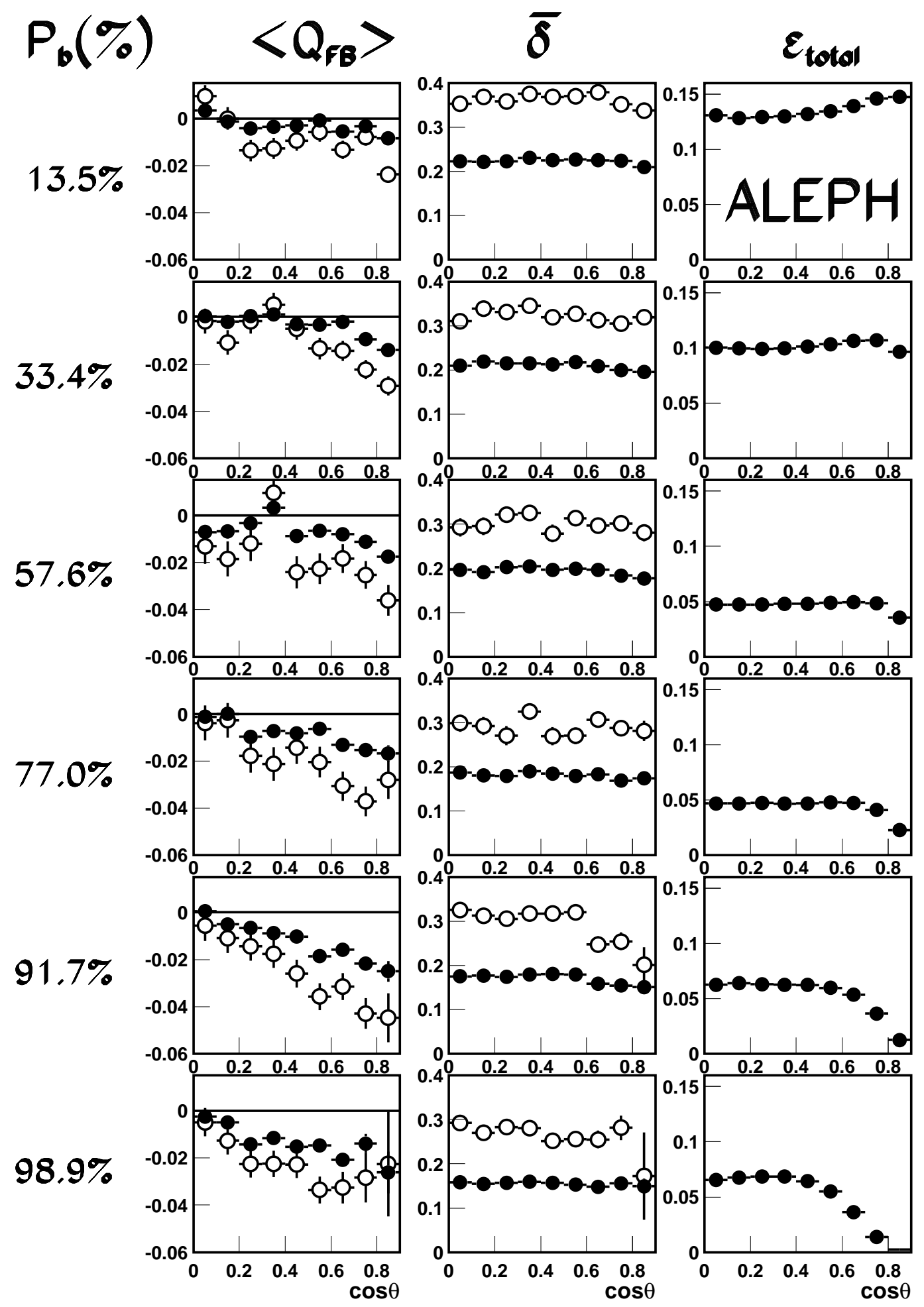

Figure 1: Measured $\left\langle Q_{\mathrm{FB}}\right\rangle$ (black dots represent $\kappa=0.5$ and open circles are used for $\kappa=2.0$ ), $\bar{\delta}$ and $\varepsilon_{\text {total }}$ distributions in each $P_{\mathrm{hem}}^{\mathrm{min}}$ bin using all peak $(\sqrt{\mathrm{s}}=91.25 \mathrm{GeV})$ data. Statistical errors only are shown. 


\begin{tabular}{|l|c|c|c|c|c|}
\hline \multirow{2}{*}{ Input } & \multicolumn{5}{|c|}{$\kappa$} \\
\cline { 2 - 6 } & 0.3 & 0.5 & 1.0 & 2.0 & $\infty$ \\
\hline$k_{u}$ & $15.3 \pm 2.5$ & $8.6 \pm 1.1$ & $5.5 \pm 1.2$ & $5.3 \pm 1.0$ & $5.8 \pm 1.3$ \\
$k_{d}$ & $30.8 \pm 4.4$ & $14.8 \pm 3.3$ & $4.7 \pm 1.9$ & $3.7 \pm 1.8$ & $4.7 \pm 3.8$ \\
$k_{s}$ & $26.6 \pm 3.5$ & $11.6 \pm 2.3$ & $5.4 \pm 1.1$ & $4.9 \pm 1.2$ & $5.1 \pm 1.8$ \\
$k_{c}$ & $15.4 \pm 2.7$ & $8.5 \pm 2.5$ & $2.2 \pm 1.1$ & $1.2 \pm 3.6$ & $0.0 \pm 8.8$ \\
$k_{b}$ & $36.4 \pm 3.6$ & $18.4 \pm 2.3$ & $8.9 \pm 1.1$ & $8.1 \pm 1.8$ & $6.5 \pm 3.6$ \\
\hline$\delta_{u}$ & $0.238 \pm 0.006$ & $0.291 \pm 0.007$ & $0.406 \pm 0.009$ & $0.528 \pm 0.012$ & $0.621 \pm 0.013$ \\
$\delta_{d}$ & $-0.143 \pm 0.005$ & $-0.171 \pm 0.007$ & $-0.229 \pm 0.011$ & $-0.289 \pm 0.016$ & $-0.339 \pm 0.019$ \\
$\delta_{s}$ & $-0.171 \pm 0.002$ & $-0.218 \pm 0.003$ & $-0.329 \pm 0.005$ & $-0.455 \pm 0.009$ & $-0.557 \pm 0.016$ \\
$\delta_{c}$ & $0.192 \pm 0.009$ & $0.200 \pm 0.010$ & $0.211 \pm 0.016$ & $0.208 \pm 0.026$ & $0.194 \pm 0.035$ \\
\hline
\end{tabular}

Table 4: Monte Carlo hemisphere charge correlations (in \%) and light quark and charm hemisphere charge separations and their systematic errors for each $\kappa$. The values are integrated over 0.0 to 0.9 in $\cos \theta$.

that systematic uncertainties enter from the physics simulation of charm and light quark tagging efficiencies.

Figure 2 shows the distributions of the extracted quantites, $\delta_{b}, \mathcal{P}_{b}$ and the value of $A_{\mathrm{FB}}^{b}$, obtained from fits to the corresponding data distributions in each $P_{\mathrm{hem}}^{\min }$ bin. The decrease in $\left|\delta_{b}\right|$ at high $\cos \theta$ reflects the observed trend in the measured $\bar{\delta}$ distributions where track losses in the beam pipe region reduce the degree of parent quark charge retention for jets at low angles. At low $b$ purities the $b$ quark rejection is most effective in the central region of the detector. The marked increase in $b$ purity at high $\cos \theta$ in the small $P_{\text {hem }}^{\min }$ (high $b$ purity) plots is due to the loss of tracks at the edge of the vertex detector acceptance. This affects $b$ events the least, as many tracks with large $p_{\mathrm{T}}$ relative to the thrust axis direction continue to tag the event. As charm and light quark events have relatively few tracks with significant impact parameters, which also tend to be more collinear with the thrust axis, these events are less likely to be tagged once the axis lies outside the VDET. As a result, the trend of increasing $b$ purity with polar angle starts at the edge of the second VDET layer close to $\cos \theta \approx 0.7$ and increases rapidly out to the edge of the second silicon layer at $\cos \theta \approx 0.9$. The fitted values of $A_{\mathrm{FB}}^{b}$ at the peak $(\sqrt{s}=91.25 \mathrm{GeV})$ are also shown in Figure 2 together with their statistical uncertainties.

Figure 3 shows the fitted $A_{\mathrm{FB}}^{b}$ values and statistical errors for each $P_{\mathrm{hem}}^{\min }$ bin. Systematic errors on the fitted values are determined from the following sources for each $\left(P_{\mathrm{hem}}^{\min }, \cos \theta\right)$ bin :

- Monte Carlo statistics - This error is due to the finite size of the simulated event samples used to estimate correction factors for the hemisphere charge correlations, corrections which take into account the $P_{\mathrm{hem}}^{\mathrm{min}}$ and $\cos \theta$ dependence of the charge separations and statistical errors from estimates of charm and light quark tagging efficiencies.

- Charm and light quark tagging efficiencies - These are calculated in a similar manner to [12] and can be broken down into contributions arising from inadequacies in the simulation of the detector and from knowledge of physics inputs in charm and light quark decays. The error is determined by varying each of the charm and light quark, detector and physics parameters, and studying the effect on the event tag efficiency. Systematic errors are evaluated by changing each of the inputs by its uncertainty in all $P_{\text {hem }}^{\min }$ bins simultaneously, thus taking into account the $100 \%$ correlation between input values for each bin.

- Charm and light quark hemisphere charge separations - These uncertainties are determined from the combined errors given in [7] and their corresponding correlation matrix. 

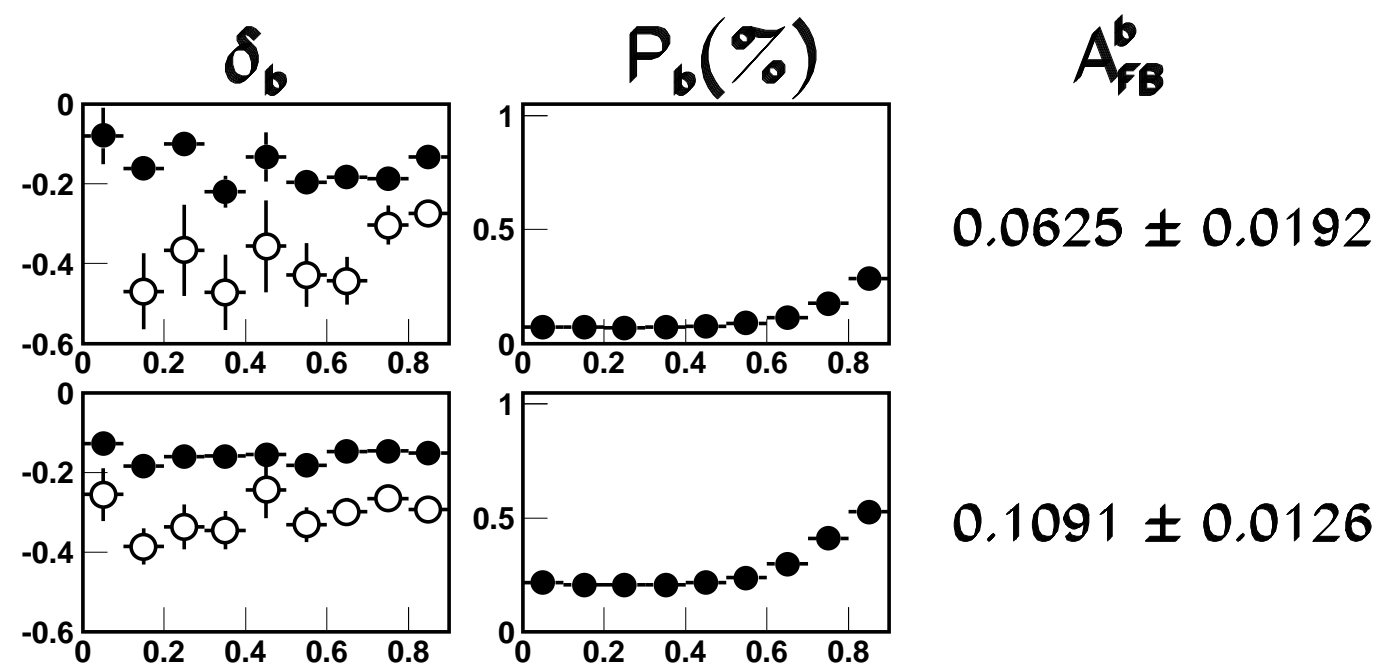

$0.1091 \pm 0.0126$
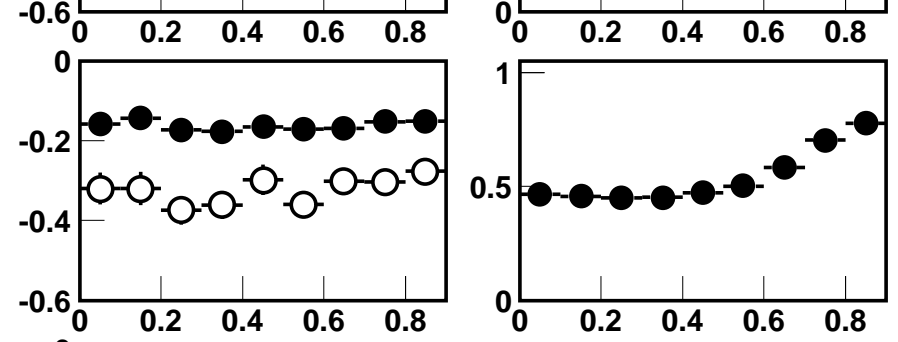

$0.1021 \pm 0.0094$
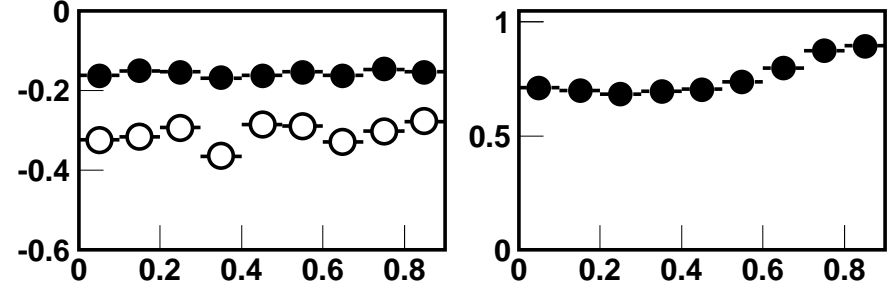

$0.0935 \pm 0.0078$
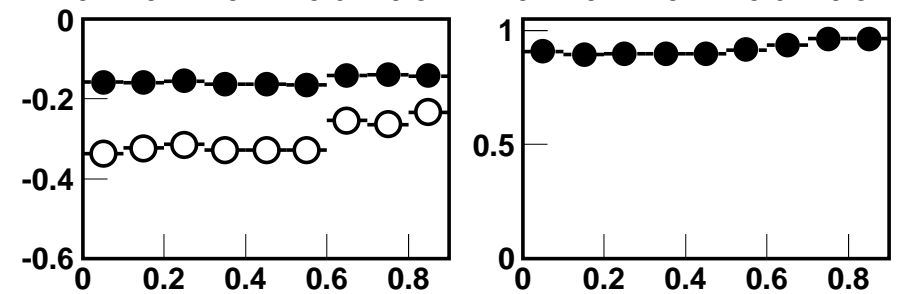

$0.1041 \pm 0.0063$
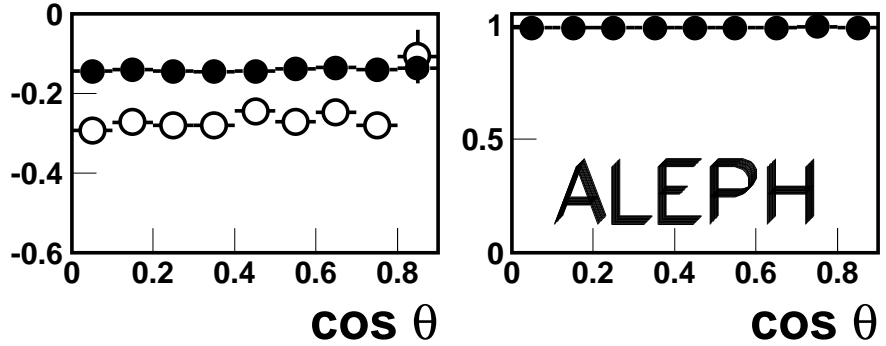

$0.1115 \pm 0.0068$

Figure 2: The extracted fit variables $\delta_{b}$ (black dots represent $\kappa=0.5$ and open circles are used for $\kappa=2.0), \mathcal{P}_{b}$ and $A_{\mathrm{FB}}^{b}$ in each $P_{\mathrm{hem}}^{\mathrm{min}}$ bin using all peak $(\sqrt{s}=91.25 \mathrm{GeV})$ data. The vertical ordering of the plots is the same as in Figure 1. 
- Hemisphere charge correlation correction Factors - These are also taken from [7] together with their estimated statistical and systematic errors due to fragmentation uncertainties.

Table 5 summarises the statistical and systematic errors on $A_{\mathrm{FB}}^{b}$. The total error on $A_{\mathrm{FB}}^{b}$ is

\begin{tabular}{|l|c|}
\hline Error Source & $\Delta A_{\mathrm{FB}}^{b}$ \\
\hline Monte Carlo statistics & 0.0006 \\
\hline$\Delta \delta^{c}$ & 0.0010 \\
$\Delta \delta^{u}, \Delta \delta^{d}, \Delta \delta^{s}$ & 0.0001 \\
\hline$k^{b}$ & 0.0019 \\
$k^{c}$ & 0.0001 \\
$\Delta k^{u}, \Delta k^{d}, \Delta k^{s}$ & 0.0000 \\
\hline$\Delta \varepsilon_{c}, \Delta \varepsilon_{u d s}$ (detector simulation) & 0.0021 \\
$\Delta \varepsilon_{c}, \Delta \varepsilon_{u d s}$ (physics input) & 0.0008 \\
\hline \hline Total Systematic Error & 0.0032 \\
\hline Statistical Error & 0.0040 \\
\hline Total Error & 0.0051 \\
\hline
\end{tabular}

Table 5: Systematic and statistical uncertainties on the value of $A_{\mathrm{FB}}^{b}$ extracted at the peak $(\sqrt{s}=91.25 \mathrm{GeV})$.

minimised when the three highest $P_{\mathrm{hem}}^{\mathrm{min}}$ bins are combined. The dominant systematic error is due to the $\cos \theta$ dependence, induced by the detector, on the background tag efficiencies, rather than on their integrated values shown in Table 3 . The remaining systematic errors are due to fragmentation uncertainties on the hemisphere correlation corrections, when extracting $\delta_{b}$ from $\bar{\delta}$, and the charm charge separation, which is limited partially by the small size of the ALEPH $D^{*}$ sample in [7]. As a consequence, the 3 highest $P_{\text {hem }}^{\min }$ bins are used to give final results.

Experimental systematic effects on the measured asymmetry in these bins are studied in [2]. Differences between positive and negative tracks are typically less than $1.5 \%$ and therefore the effect on $\left\langle Q_{\mathrm{FB}}\right\rangle$ is negligible. Uncertainties due to secondary interactions in the material of ALEPH are determined with the aid of photon conversions, which indicate a forward-backward material asymmetry of $0.036 \pm 0.010 \%$ [7]. This is multiplied by the total charge, $\langle Q\rangle=$ $\left\langle Q_{\mathrm{F}}+Q_{\mathrm{B}}\right\rangle$ in the 3 highest $P_{\mathrm{hem}}^{\min }$ bins, to give a systematic uncertainty on $\left\langle Q_{\mathrm{FB}}\right\rangle$ of $0.06 \%$, and hence on $A_{\mathrm{FB}}^{b}$.

Combining the results from the 3 highest $P_{\text {hem }}^{\min }$ bins, using data collected close to the $\mathrm{Z}$ peak, the $b$ quark asymmetry is determined to be :

$$
\left.A_{\mathrm{FB}}^{b}(\sqrt{s}=91.25 \mathrm{GeV})=0.1040 \pm 0.0040 \text { (stat. }\right) \pm 0.0032 \text { (syst.) } .
$$

To determine the effect of the fitting procedure in bins of $\left(\cos \theta, P_{\mathrm{hem}}^{\min }\right)$, the determination of $A_{\mathrm{FB}}^{b}(\sqrt{s}=91.25 \mathrm{GeV})$ is repeated using equation (12) in a single bin of $\cos \theta$ from $0.0 \rightarrow 0.9$, $P_{\text {hem }}^{\text {min }}$ from $0.0 \rightarrow 0.001$ and a $\kappa$ of 0.5 . The central value remains consistent with the full fit and the statistical error increases by about 10\%. It is also verified that repeating the fit for individual $\kappa$ values leads to statistically consistent results. The fitted off-peak values of $A_{\mathrm{FB}}^{b}$ are

$$
\begin{aligned}
& A_{\mathrm{FB}}^{b}(\sqrt{s}=89.43 \mathrm{GeV})=0.0746 \pm 0.0178(\text { stat. }), \\
& A_{\mathrm{FB}}^{b}(\sqrt{s}=92.97 \mathrm{GeV})=0.0924 \pm 0.0179(\text { stat. }) .
\end{aligned}
$$

The variation of $A_{\mathrm{FB}}^{b}$ with centre-of-mass energy is shown in Figure 4. Assuming the Standard Model energy dependence of $A_{\mathrm{FB}}^{b}$ with $\sqrt{s}$, on and off peak data are combined to obtain a final value for the observed asymmetry at the $\mathrm{Z}$ mass $\left(\sqrt{s}=m_{\mathrm{Z}}=91.1866 \mathrm{GeV}\right)$ of :

$$
A_{\mathrm{FB}}^{b}\left(\sqrt{s}=m_{\mathrm{Z}}\right)=0.1017 \pm 0.0038 \text { (stat.) } \pm 0.0032 \text { (syst.). }
$$




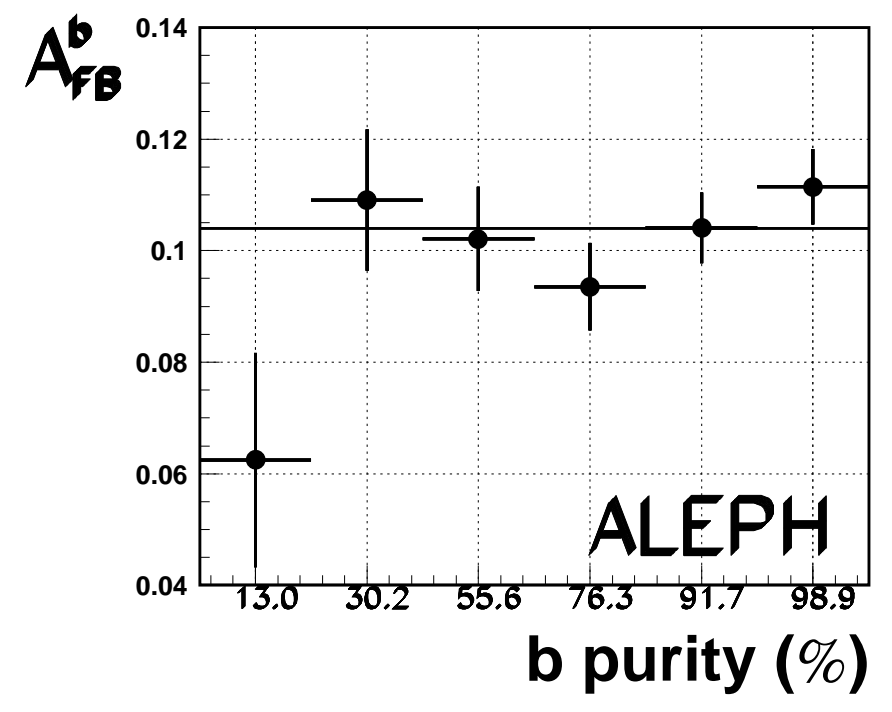

Figure 3: Fitted $A_{\mathrm{FB}}^{b}$ values and statistical errors for each $P_{\mathrm{hem}}^{\min }$ bin using all peak data. The line represents the measured peak value of $A_{\mathrm{FB}}^{b}=0.1040$.

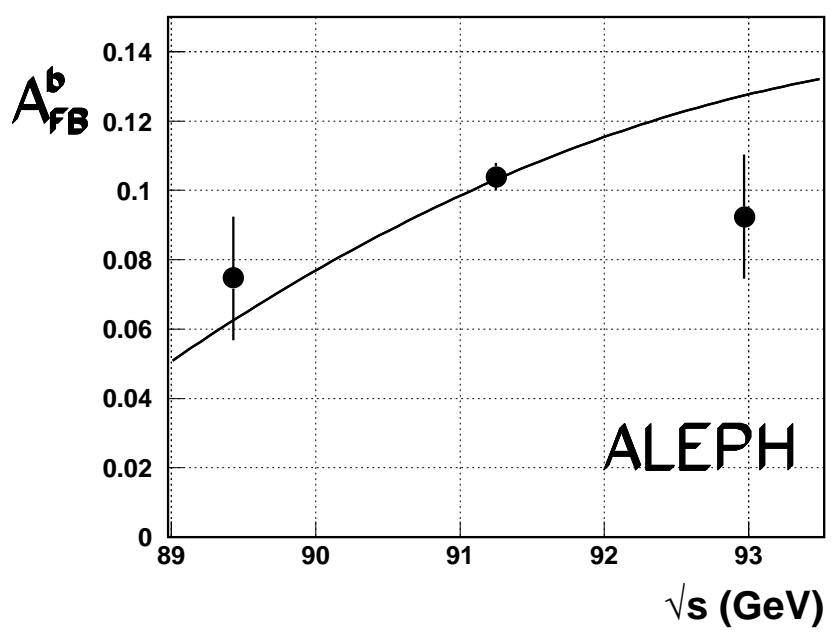

Figure 4: The energy $(\sqrt{s})$ dependence of the measured $A_{\mathrm{FB}}^{b}$ values combined into three energy points. The errors shown are statistical only and the curve represents the Standard Model prediction corresponding to the fitted asymmetry, $A_{\mathrm{FB}}^{b}\left(\sqrt{s}=m_{\mathrm{Z}}\right)=0.1017$. 
A general procedure to correct the measured asymmetry, $A_{\mathrm{FB}}^{b}$, for QED and QCD effects is described in [16]. In this analysis, the QCD corrections are unnecessary for the following reason. The $b$-quark charge separation, $\delta_{b}$, is defined in the Monte Carlo with respect to the original $b \bar{b}$ quark pair orientation, prior to gluon or final state photon radiation, parton shower, hadronisation and $B^{0} \bar{B}^{0}$ mixing. All these effects are therefore included, by construction, in the analysis, as far as they are properly modelled in the JETSET [14] hadronisation model. The $\delta_{f}$ quantities define the relationship between the measurable jet charge asymmetry and the electroweak asymmetry, whereas the correlation factors, $k_{f}$, define the relationship between the measurable $\bar{\delta}$ and $\delta_{f}$.

The only remaining corrections applied, to relate the measured asymmetry to the electroweak asymmetry, $A_{\mathrm{FB}}^{0, b}$, and neutral current couplings, are those for photon exchange diagrams, ISR radiation and the fact that the centre-of-mass energy is not exactly that of the $\mathrm{Z}$ pole.

In the Standard Model, the dependence of all asymmetries upon electroweak radiative corrections of interest is embedded in the variation of the electroweak mixing angle, $\sin ^{2} \theta_{\mathrm{W}}^{\mathrm{eff}}$. This is also true of all light quark partial widths. The electroweak aymmetry, $A_{\mathrm{FB}}^{0, b}$, is obtained by varying the values of the lighter quark asymmetries and all the quark branching ratios consistently with the $b$-quark asymmetry, according to the sensitivities given in Table 2.

Combining both statistical and systematic uncertainties, the corrected electroweak asymmetry is then given by :

$$
A_{\mathrm{FB}}^{0, b}=0.1056 \pm 0.0054,
$$

from which the effective weak mixing angle is determined to be :

$$
\sin ^{2} \theta_{\mathrm{W}}^{\mathrm{eff}}=0.23109 \pm 0.00096 .
$$

\section{Summary and Conclusions}

In a data sample of 4.1 million hadronic $\mathrm{Z}$ decays recorded with the ALEPH detector at LEP, from 1991 to 1995, the observed forward-backward hemisphere charge asymmetry is analysed in order to determine the $b$ forward-backward asymmetry, $A_{\mathrm{FB}}^{b}$, and the effective weak mixing

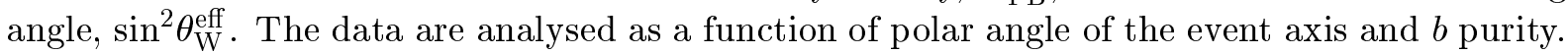
Combining on and off peak data around the $\mathrm{Z}$ resonance, the measured asymmetry at $\sqrt{s}=m_{\mathrm{Z}}$ is determined to be :

$$
A_{\mathrm{FB}}^{b}\left(\sqrt{s}=m_{\mathrm{Z}}\right)=0.1017 \pm 0.0038 \text { (stat.) } \pm 0.0032 \text { (syst.). }
$$

Combining both statistical and systematic uncertainties, and applying corrections for QED, photon exchange and dependencies on Standard Model input parameters, the corrected electroweak asymmetry, $A_{\mathrm{FB}}^{0, b}$, and effective weak mixing angle are given by :

$$
A_{\mathrm{FB}}^{0, b}=0.1056 \pm 0.0054,
$$

and :

$$
\sin ^{2} \theta_{\mathrm{W}}^{\mathrm{eff}}=0.23109 \pm 0.00096 .
$$

The dominant systematic uncertainties on this measurement are due to residual discrepancies in the tagging efficiencies found between detector simulation and data and, to a lesser extent, from fragmentation uncertainies on the correlations between forward and backward hemisphere charges.

The values are in agreement with those of the ALEPH high $p_{\mathrm{T}}$ lepton analysis[15] and statistical and systematic errors are almost entirely uncorrelated. The combined results are :

$$
\begin{array}{rll}
A_{\mathrm{FB}}^{0, b} & =0.1031 & \pm 0.0037, \\
\sin ^{2} \theta_{\mathrm{W}}^{\text {eff }}= & 0.23156 & \pm 0.00066 .
\end{array}
$$




\section{Acknowledgements}

We wish to thank our colleagues in the CERN accelerator divisions for the excellent performance of the LEP machine. Thanks are also due to the engineers and technicians at all the collaborating institutions for their contribution to the success of ALEPH. Those of us from non-member states are grateful to CERN for its hospitality.

\section{References}

[1] S. Glashow, Nucl. Phys. 20 (1961) 579; A. Salam, in Elementary Particle Theory, ed. N. Svartholm, (1968); S. Weinberg, Phys. Rev. Lett. 19 (1967) 1264.

[2] ALEPH Collaboration, "Measurement of $A_{\mathrm{FB}}^{b}$ in Lifetime-Tagged Heavy Flavour Decays" Phys. Lett. B335 (1994) 99.

[3] DELPHI Collaboration, "Measurement of the Forward-Backward Asymmetry of $e^{+} e^{-} \rightarrow$ $Z \rightarrow b \bar{b}$ using Prompt Leptons and a Lifetime Tag", Z. Phys. C65 (1994) 569.

[4] OPAL Collaboration, "Measurements of the b Quark Forward-backward Asymmetry around the $Z^{0}$ Peak Using Jet Charge and Vertex Charge”, Z. Phys. C75 (1997) 385.

[5] ALEPH Collaboration, "A Precise Measurement of $\Gamma(Z \rightarrow b \bar{b}) / \Gamma(Z \rightarrow$ hadrons $)$ ", Phys. Lett. B313 (1993) 535.

[6] ALEPH Collaboration, "Measurement of the Hadronic Charge Asymmetry in Hadronic Z decays", Phys. Lett. B259 (1991) 377.

[7] ALEPH Collaboration, "Determination of $\sin ^{2} \theta_{\mathrm{W}}^{\mathrm{eff}}$ using Jet Charge Measurements in Hadronic Z Decays", Z. Phys. C71 (1996) 357.

[8] R. Field and R. Feynman, Nucl. Phys. B136 (1978) 1.

[9] ALEPH Collaboration, "ALEPH: A Detector for Electron-Positron Annihilations at LEP", Nucl. Inst. and Methods, A294 (1990) 121.

[10] ALEPH Collaboration, "Performance of the ALEPH Detector at LEP", Nucl. Inst. and Methods, A360 (1995) 481.

[11] The LEP Collaborations, ALEPH, DELPHI, L3, OPAL and the Electroweak Working Group and SLD Heavy Flavour Working Group, "A Combination of Preliminary Electroweak Measurements and Constraints on the Standard Model", CERN-PPE/97-154.

[12] ALEPH Collaboration, "A measurement of $R_{b}$ using a lifetime-mass tag", Phys. Lett. B401 (1997) 150.

[13] ALEPH Collaboration, "Heavy Flavour Production and Decay with Prompt Leptons in the ALEPH detector", Z. Phys. C62 (1994) 179.

[14] T. Sjöstrand, CERN TH 7112-93. T. Sjöstrand, Computer Physics Commun. 39 (1986) 347. T. Sjöstrand and M. Bengtsson, Computer Physics Commun. 43 (1987) 367.

[15] ALEPH Collaboration, "Measurement of the b Forward-backward Asymmetry and Mixing using High-p $p_{\mathrm{T}}$ Leptons", Phys. Lett. B384 (1996) 414.

[16] The LEP Experiments : ALEPH, DELPHI, L3 and OPAL, "Combining Heavy Flavour Electroweak Measurements at LEP", Nucl. Instr. and Methods A378 (1996) 101. 